\title{
Validation of Tissue Microarrays Using P53 Immunohistochemical Studies of Squamous Cell Carcinoma of the Larynx
}

\author{
Mark C. Griffin, M.D., Robert A. Robinson, M.D., Douglas K. Trask, M.D., Ph.D. \\ Department of Otolaryngology/Head and Neck Surgery (MCG, DKT) and Department of Pathology (RAR), \\ University of Iowa Health Care, Iowa City, Iowa
}

Tissue microarrays are a powerful new tissueconserving technology in the study of cancer, allowing simultaneous study of a large number of tumor specimens. We sought to ascertain the utility of tissue microarrays in head and neck cancer pathology using squamous cell carcinoma of the larynx as a model system. Whole-specimen slides from 44 different laryngeal squamous cell carcinomas were stained for p53 expression. Microarrays were then generated by taking six $0.6-\mathrm{mm}$ core biopsies from each of the 44 specimens. The whole sections and the microarrays were independently scored for p53 expression. Twenty-three (53\%) of the 44 tumor specimens were positive for p53. Forty-four of the 264 core biopsies $(17 \%)$ were not given a score because of the lack of tumor cells. Seventy-eight percent of the individual discs on the microarray had scores in agreement with those of the whole-section slides. Among biopsy discs with tumor cells present, 94.5\% were in agreement with the whole-section slide. The average probability that four randomly chosen biopsy discs, considered together, would accurately identify the presence of p53 staining in a whole section was 0.97 (95\% CI .93-1.0). We conclude that tissue microarrays for squamous cell carcinomas can accurately represent immunohistochemical results of whole-slide specimens when four or more samples are used. Tissue microarrays are an important technique that may be applied to immunohistochemical studies of head and neck cancer.

Copyright (C) 2003 by The United States and Canadian Academy of Pathology, Inc.

VOL. 16, NO. 12, P. 1181, 2003 Printed in the U.S.A.

Date of acceptance: August 15, 2003.

Supported in part by the Frederick W. Stamler Professorship (RAR). Address reprint requests to: Douglas K. Trask, M.D., Ph.D., Department of Otolaryngology/Head and Neck Surgery, University of Iowa Hospitals and Clinics, 200 Hawkins Drive, Iowa City, IA 52242; fax: 319-356-4547; e-mail: douglas-trask@uiowa.edu.

DOI: 10.1097/01.MP.0000097284.40421.D6
KEY WORDS: Immunohistochemistry, Laryngeal neoplasms, p53, Squamous cell carcinoma, Tissue microarray.

Mod Pathol 2003;16(12):1181-1188

Tissue microarray analysis (TMA) is a tissueconserving technology that has been applied to the study of multiple cancers. The technique, invented by Wan et al. (1), involves taking biopsy "cores" from multiple paraffin-embedded tissue specimens and inserting them into a single paraffin receiver block. Kononen et al. (2) popularized microarray technology by describing a single paraffin receiver block that contains $\leq 1000$ different yet uniformlysized tissue samples. These blocks then produce many slides for experimentation.

This technology offers several advantages. A large number of tumor samples may be studied simultaneously under identical conditions in the laboratory, reducing lab error (3). Efficiency is also maximized, in that a few slides generate the data that would otherwise require hundreds, or even thousands, of slides to obtain. The tissue in the microarrays may be studied in a variety of ways, including immunohistochemical analysis, fluorescent in situ hybridization, and laser microdissection for biochemical analysis (4). The technique dramatically conserves reagents, tissue samples, and time. It has successfully been used to develop institutionspecific staining profiles for various antibodies as a powerful quality assurance analysis (5).

The main criticism of tissue microarrays is that the amount of tissue analyzed with this technique may not be representative of the whole specimen. This could be especially problematic in epithelial tumors, where intratumor heterogeneity has been shown to exist $(6,7)$. Validation experiments have been performed to support the use of tissue microarray technology in various epithelial tumors. In studying breast carcinoma, Camp et al. (8) suggested that two discs $0.6 \mathrm{~mm}$ in diameter accurately represent whole-section specimens $95 \%$ of the time 
in immunohistochemical studies. Hoos et al. (9) studied various fibroblastic tumors and recommended using three discs to achieve a $96-98 \%$ concordance between microarray and whole-tissue slides when performing immunohistochemical analysis. Fernebro et al. (10) evaluated the technique in rectal cancer and found good correlation when three discs were taken. Others have validated this technology with malignant fibrous histiocytoma (11).

An estimated 10,000 new cases of carcinoma of the larynx are diagnosed each year in the United States, resulting in an estimated 4,000 deaths in 2001 (12). P53 overexpression has been the subject of much interest in carcinoma of the larynx. Bradford et al. (13) demonstrated that p53 overexpression in advanced laryngeal cancers predicted organ preservation with induction chemotherapy and radiation but do not show a difference in survival. In that study, $61 \%$ of the specimens were positive for p53 overexpression. It is also important to note that p53 expression has been shown to be heterogeneous within individual laryngeal tumor specimens (14).

In the current study, we have generated tissue microarrays of squamous cell carcinomas of the larynx. We used p53 because it has been well characterized in head and neck carcinomas and because it has heterogeneous intertumor and intratumor staining properties. The goal of the study is to validate tissue microarray analysis in squamous cell carcinoma of the larynx.

\section{MATERIALS AND METHODS}

\section{Patient Population}

Patients diagnosed with laryngeal carcinoma between 1991 and 1996 were identified from the head and neck tumor registry at the University of Iowa. Only patients who underwent primary surgical excision of their tumors at the University of Iowa were included. Patients were excluded from the study if they had received radiation therapy to the head and neck or any form of chemotherapy before their laryngeal tumor excision. Patients were also excluded from the study if the primary surgical specimen was not available. Paraffin-embedded tissue blocks were selected for each included subject.

\section{Microarray Generation}

Slides were prepared by sectioning each tissue block using a microtome. One slide from each specimen was stained for p53 expression. After the whole-block sections were made, tissue microarrays were designed to include six different $0.6-\mathrm{mm}$ core biopsies from each paraffin tissue block. Biop- sies were selected from areas of the paraffin tissue block that grossly appeared to contain viable tumor. Two different microarrays, each with approximately 140 core biopsies, were generated using a microarray instrument (Beecher Instruments, Inc., Sun Prairie, WI). Once made, the microarrays were placed upside-down on a glass slide and baked for 30 minutes at $37^{\circ} \mathrm{C}$ to even out the surface. Five micron sections were prepared.

\section{Immunohistochemical Analysis}

Each whole-section slide was stained for p53 expression using the LSAB II kit obtained from DAKO (Carpenteria, CA). Briefly, sections were deparaffinized in xylenes and rehydrated in graded alcohols. Antigen retrieval was accomplished with citrate buffer ( $\mathrm{pH}$ 6.0) and microwave heating. Slides were allowed to cool to room temperature for 20 minutes. Endogenous peroxidase activity was quenched using $1.88 \% \mathrm{H}_{2} \mathrm{O}_{2}$ in distilled water for 8 minutes. P53-D07 (DAKO) primary antibody, used at a dilution of 1:50, was applied, and slides were incubated for 1 hour at room temperature. Secondary universal antibody was applied for 20 minutes at room temperature, rinsed, and then covered with the streptavidin label for 20 minutes at room temperature. Chromagraphic precipitation was accomplished with 3,3'-diaminobenzidine chromogen. A counterstain was performed with $10 \%$ Harris Hematoxylin for 3 minutes. Negative control slides were prepared by substituting normal mouse serum for primary antibody. A colon adenocarcinoma that overexpressed p53 was used as a positive control.

Only strong nuclear staining, as comparable to that seen in the colonic adenocarcinoma control, was considered positive. The authors scored the slides independently in a blinded manner. Each whole specimen was given a score from 0 to 4 based on the proportion of tumor cells with staining: 0 represents $<2 \%$ of the tumor cells staining positive, 1 represents $2-25 \%, 2$ represents $26-50 \%$, 3 represents $51-75 \%$, and 4 represents $76-100 \%$ staining of tumor cells. The scores from each author were averaged to obtain the average score for that specimen.

Scoring of the microarrays was performed similarly. Each disc was scored separately in a blinded manner, from 0 to 4 , representing the proportion of tumor cells with staining as described above for the whole-tissue stains. Some discs had no tumor cells sampled and were scored in one of the following three manners: necrotic tumor cells only, nontumor cells only, or no cells present (i.e., the disc was absent). 


\section{Data Analysis}

The whole-section scores from the three evaluators were averaged to yield the standard score for each specimen. Each of the six biopsy discs also received an average score. A disc was considered p53 positive if it received an average score $\geq 0.67$. True-negative biopsy discs were those with average scores of $0-0.3$, and that were taken from whole specimens with a score of 0 . True positives were defined as having an average disc score of $\geq 0.67$ and were taken from whole specimens with a nonzero score. The predictive value for a single disc was calculated by adding true negatives and true positives, divided by the total number of discs, that is, 264.

Probabilities were calculated to estimate the chance that one, two, three, four, five, or six randomly chosen biopsy discs would correctly identify positive or negative staining in the whole-tissue specimen. The following assumptions were made to make these calculations. If two discs were received, one showing p53 staining and one not showing staining, then the two samples were assumed to represent a whole specimen with positive p53 staining. This assumption was made, in part, because greater importance was placed on avoiding false negatives than on avoiding false positives. Also, it is more likely that a whole specimen positive for p53 would produce a disc without p53 staining (because of staining heterogeneity) than a whole specimen negative for p53 would produce a disc positive for p53. Likewise, if three discs were randomly taken, and one showed staining, one showed no staining, and one did not contain tumor cells, these discs were assumed to represent a whole specimen with positive p53 staining. However, if two discs showed no staining and one showed staining, the whole specimen was assumed to have no staining. Similar assumptions were made for 4 , 5 , and 6 discs. Using these assumptions, the probabilities were calculated based on the hypergeometric distribution method (15).

\section{RESULTS}

\section{Patient Demographics}

Age, sex, subsite, and tumor stage data are summarized in Table 1 . Twenty-nine $(66 \%)$ of the patients were men, 15 (44\%) were women. Eleven (25\%) were aged $<50$ years, 24 (55\%) were between the ages of 50 and 65 years, and $9(20 \%)$ were aged $>65$ years at the time of diagnosis. Four $(9 \%)$ had carcinoma of the glottis, and 40 (91\%) had supraglottic carcinoma. Pathologic tumor stage was T1 in 1 patient (2\%), T2 in $8(18 \%)$, T3 in $20(45 \%)$, and T4 in $15(34 \%)$. There was pathologically confirmed nodal disease in $23(52 \%)$ of the patients at the time
TABLE 1. Summary of Patient Data

\begin{tabular}{lc}
\hline & Number $(\%)$ \\
\hline Sex & $29(66)$ \\
Men & $15(34)$ \\
Women & \\
Age & $11(25)$ \\
$<50$ & $24(55)$ \\
50-65 & $9(20)$ \\
$>65$ & \\
Subsite & $40(91)$ \\
Supraglottis & $4(9)$ \\
Glottis & \\
T stage & $1(2)$ \\
T1 & $8(18)$ \\
T2 & $20(45)$ \\
T3 & $15(34)$ \\
T4 & \\
N stage & $21(47)$ \\
N0 & $7(16)$ \\
N1 & $13(30)$ \\
N2 & $3(7)$ \\
N3 & \\
AJCC stage & $4(9)$ \\
II & $16(36)$ \\
III & $24(55)$ \\
IV & \\
\hline
\end{tabular}

of resection. Using the AJCC tumor staging system, $4(9 \%)$ patients had Stage II disease, 16 (36\%) had Stage III, and $24(55 \%)$ had Stage IV. The lack of Stage I disease in this study reflects the fact that such tumors are often treated with primary external beam radiation and sufficient tissue is not available as a primarily resected specimen.

\section{Immunohistochemical Analysis}

Of 46 tumor specimens initially selected, 2 had no identifiable tumor on the whole-section slide and were omitted from analysis. Of the remaining 44,21 (48\%) were given a score of " by all three evaluators, indicating no p53 staining. Four (9\%) received an average score between 1 and 1.3, five (11\%) received an average score between 1.6 and 2.3 , five $(11 \%)$ received an average score between 2.6 and 3.3, and nine (20\%) received an average score between 3.7 and 4 .

With regard to the microarrays, 6 core biopsies from each of 44 tumor specimens produced 264 discs. Forty-four of 264 (17\%) discs were not given a score for one of three reasons: discs were altogether missing $(n=3)$, they contained only necrotic tumor cells with pyknotic nuclei $(n=13)$, or they contained no tumor cells $(n=28)$. Figures 1 and 2 show representative examples of whole tumor sections and their corresponding six core biopsies. Figure 1 demonstrates complete concordance between the whole section, scored as 4 , and the core biopsies, each scored as 4 . Two of the six core biopsies in Figure 2, however, could not be given a numerical score. The disc in Panel C (Fig. 2) did not contain 

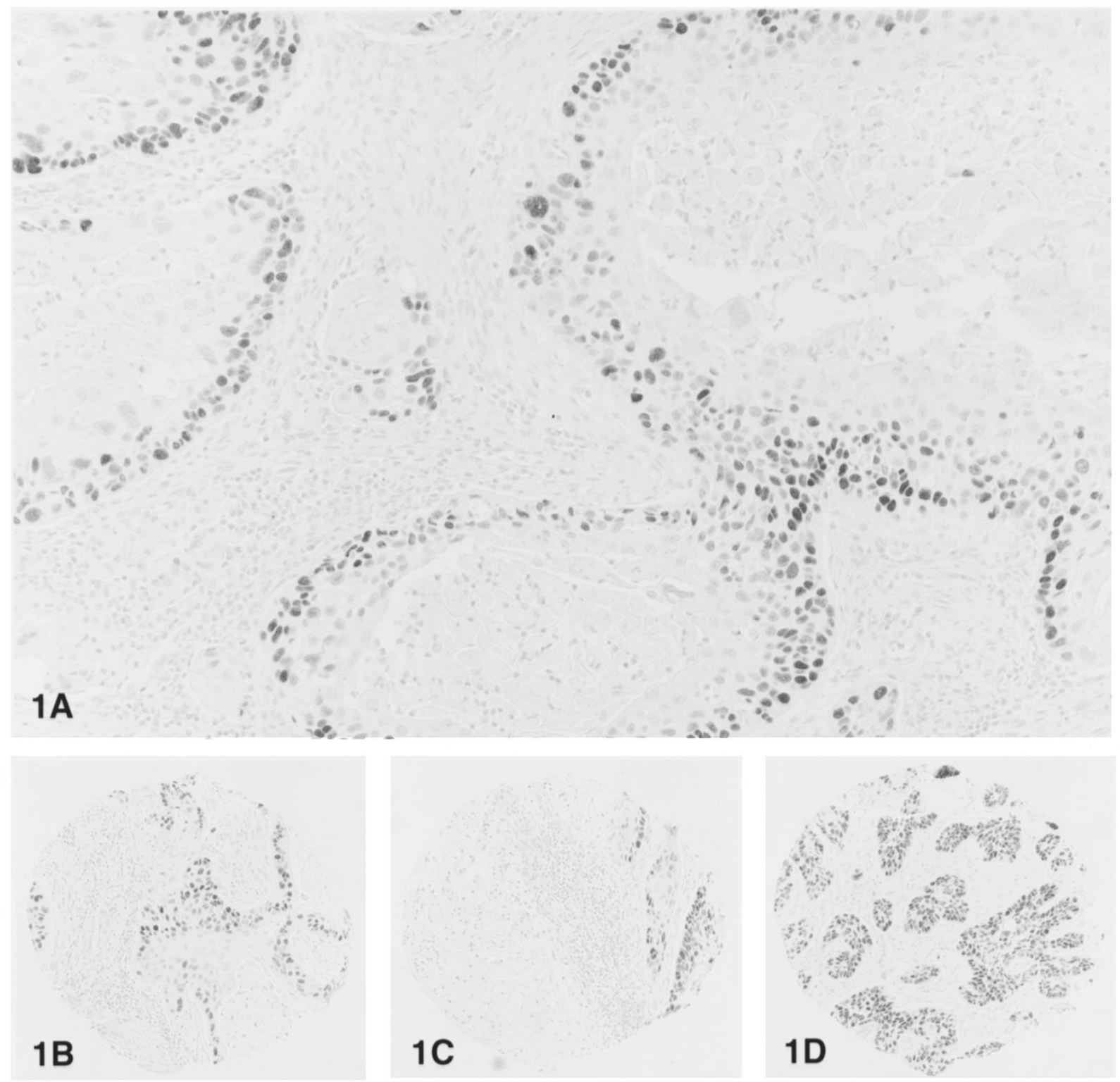

$1 \mathrm{C}$

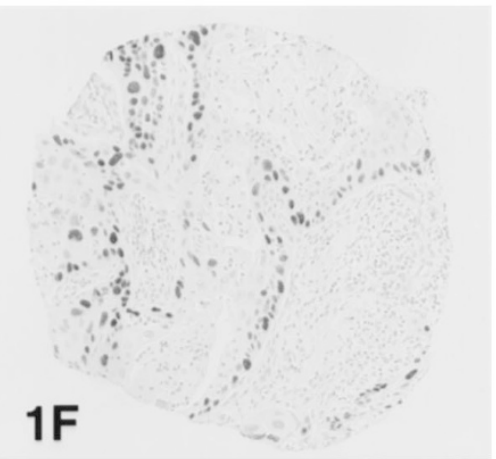

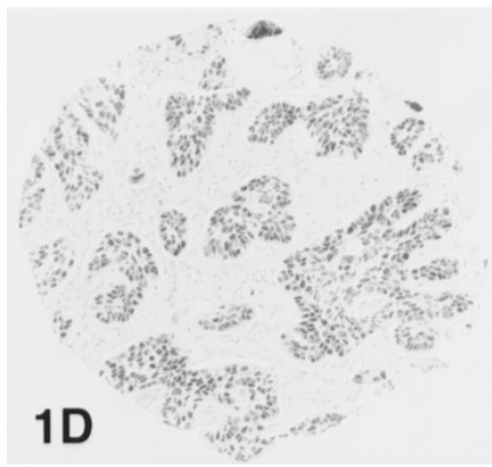

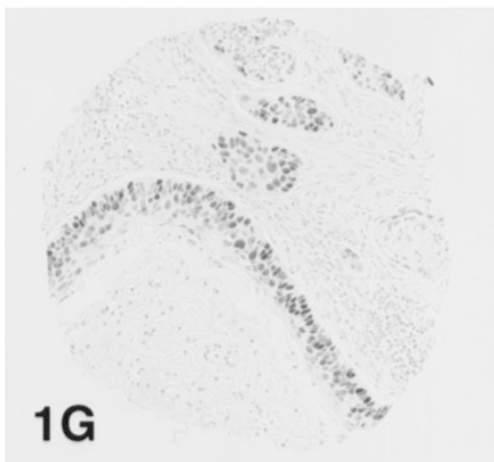

FIGURE 1. Squamous cell carcinoma of the larynx, stained for p53 (see Patient 41, Table 2). A, an area of the whole section, which received an average score of 4 , indicating that $76-100 \%$ of the tumor cells were p53 positive. B-G, the corresponding six discs taken from this specimen. Each disc also received an average score of 4.

any tumor cells, and the disc in Panel G contained only necrotic tumor cells with pyknotic nuclei.

The average scores of each patient's specimen and their six biopsy discs are displayed in Table 2.
Of biopsies from the 21 whole specimens with no p53 staining (those with a whole-section score of 0 ), 90 have an average score of 0,5 have an average score of 0.3 , and 8 have an average score between 

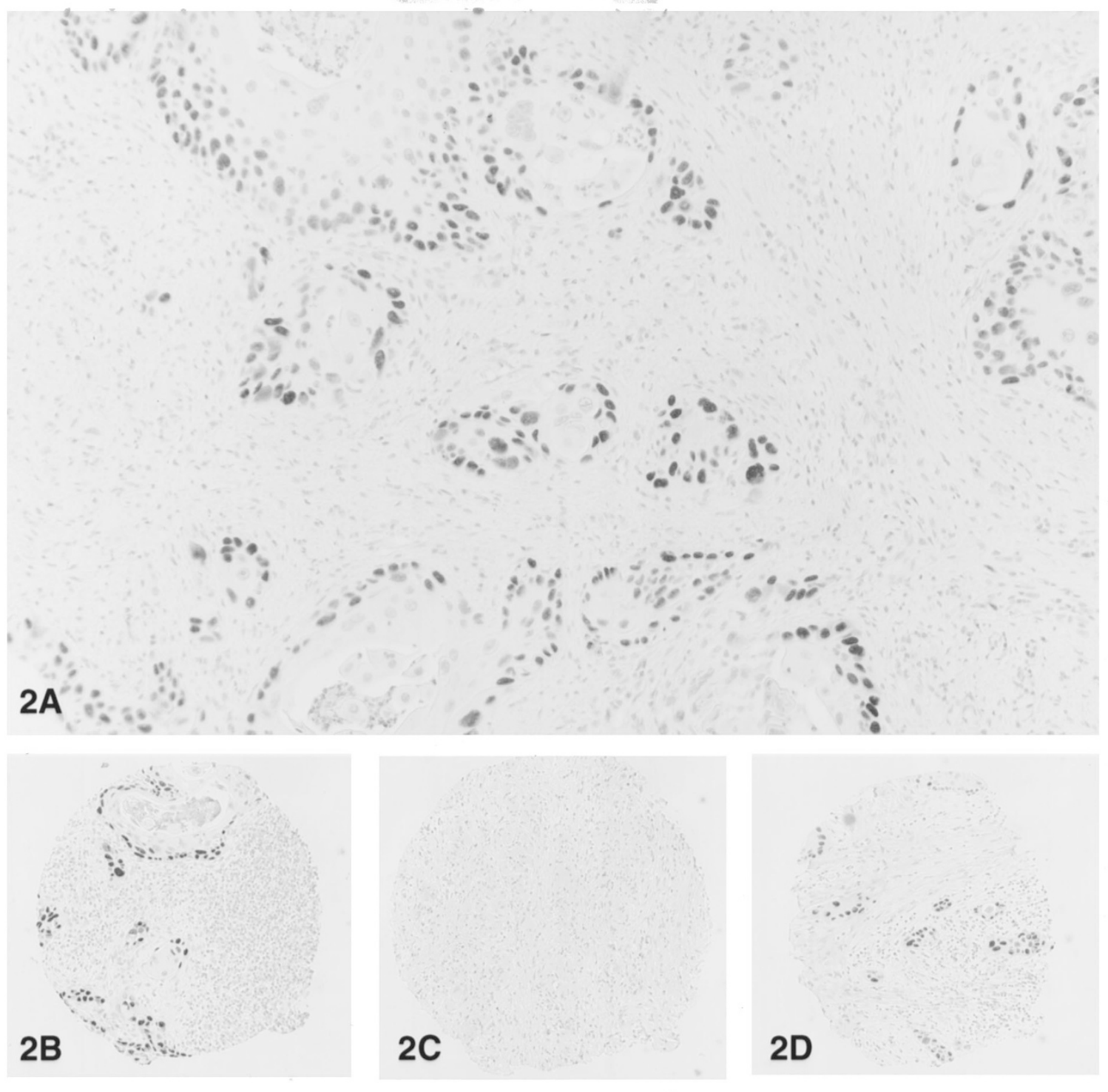

\section{C}

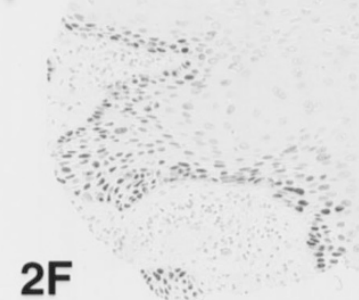

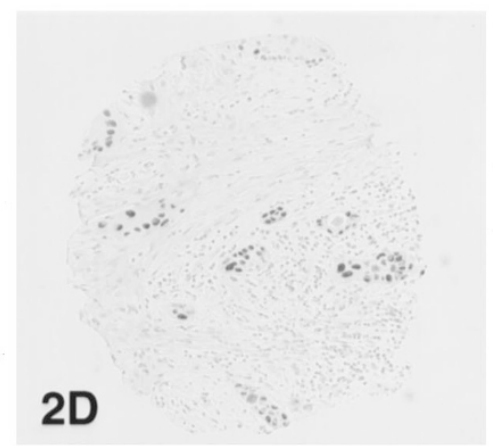

2D

\section{G}

FIGURE 2. Squamous cell carcinoma of the larynx, stained for p53 (see Patient 33, Table 2). A, an area of the whole section, which received an average score of 3, indicating that $51-75 \%$ of the tumor cells were p53 positive. B-G, the corresponding six discs taken from this specimen. Of note, the disc in Panel C was scored as NT, or not having any tumor cells present. The disc in Panel G was scored NC, or having only necrotic tumor cells present.

0.67 and 1.3. These last 8 are considered false positives, yielding a $7.8 \%$ false-positive rate. Of all the discs that were taken from specimens with no p53 staining, $95 / 126$, or $75 \%$, suggested no staining. The remaining $25 \%$ of the discs were either false positives or could not be assigned a score for the reasons mentioned above.
Twenty-three whole specimens were given scores from 1 to 4 , indicating positive p53 staining. There were three false negatives, discs taken from whole specimens with positive p53 staining but that received an average score of zero. Thus, the falsenegative rate among all discs taken from specimens with positive p53 staining is 3 in 115 or $2.6 \%$. Of all 
TABLE 2. Summary of Immunohistochemical Data

\begin{tabular}{|c|c|c|c|c|c|c|c|}
\hline \multirow{2}{*}{$\begin{array}{c}\text { Patient } \\
1\end{array}$} & \multirow{2}{*}{$\begin{array}{c}\text { Whole Section } \\
\text { Score }\end{array}$} & \multicolumn{6}{|c|}{ Average Disc Scores } \\
\hline & & 0 & 0 & 0 & 0 & 0 & 0 \\
\hline 2 & 0 & 0 & 0 & 0 & 0 & 0 & 0 \\
\hline 3 & 0 & 0 & 0 & NT & 0 & NT & 0 \\
\hline 4 & 0 & 0 & 0 & NT & MS & $\mathrm{NC}$ & NC \\
\hline 5 & 0 & 0 & 0 & 0 & 0 & MS & 0 \\
\hline 6 & 0 & NT & 1 & 0 & 0 & 0 & 0 \\
\hline 7 & 0 & 0 & 0 & 0 & 0 & 0 & 0 \\
\hline 8 & 0 & 0 & 0 & NT & 0 & 0 & 0 \\
\hline 9 & 0 & 0 & 0 & 0 & 0 & 0 & 0 \\
\hline 10 & 0 & 0 & 0 & 0 & 0 & 0 & 0 \\
\hline 11 & 0 & NT & 0 & NT & 1 & NT & 0 \\
\hline 12 & 0 & 0 & 0 & 0 & 1 & 0 & 0 \\
\hline 13 & 0 & 0 & 0 & 0 & 0 & 0 & 0 \\
\hline 14 & 0 & 1 & 1 & 0 & NT & 0 & 0 \\
\hline 15 & 0 & 0 & 1 & 0 & 0 & 0 & 0 \\
\hline 16 & 0 & 0 & 0 & 0 & 0 & 0 & 0 \\
\hline 17 & 0 & NT & 0 & 0 & NC & 0 & 0 \\
\hline 18 & 0 & NC & 0 & 0 & 0 & 0 & 0 \\
\hline 19 & 0 & 1 & 0 & 0 & NC & 0 & 1 \\
\hline 20 & 0 & 0 & NT & 0 & 0 & NT & 0 \\
\hline 21 & 0 & 0 & 0 & NT & NT & NT & NT \\
\hline 22 & 1 & NT & 1 & 0 & NT & NT & 1 \\
\hline 23 & 1 & NT & 1 & $\mathrm{NC}$ & NT & 1 & 1 \\
\hline 24 & 1.3 & 2 & 2 & NT & 2 & 3 & NT \\
\hline 25 & 1.3 & 3 & 2 & 2 & 2 & 2 & NC \\
\hline 26 & 1.7 & 0 & 2 & 2 & 3 & 2 & 2 \\
\hline 27 & 1.7 & 1 & 1 & 1 & 1 & 2 & 1 \\
\hline 28 & 2.3 & 4 & 3 & 3 & 2 & 2 & 2 \\
\hline 29 & 2.3 & 1 & 2 & 2 & 2 & 3 & 2 \\
\hline 30 & 2.3 & 3 & 1 & NT & NT & 2 & 2 \\
\hline 31 & 2.7 & 2 & 3 & 3 & NT & 4 & 4 \\
\hline 32 & 2.7 & 3 & 4 & 4 & 4 & 4 & 4 \\
\hline 33 & 3 & NC & 2 & 4 & 4 & NT & 4 \\
\hline 34 & 3 & 4 & 4 & 4 & 3 & 4 & 3 \\
\hline 35 & 3.3 & 4 & 3 & 4 & 4 & 4 & 3 \\
\hline 36 & 3.7 & 4 & 4 & 4 & 3 & 3 & 4 \\
\hline 37 & 3.7 & 4 & $\mathrm{NC}$ & $\mathrm{NC}$ & NT & 3 & 4 \\
\hline 38 & 3.7 & NT & 4 & 4 & 4 & 4 & 4 \\
\hline 39 & 3.7 & 3 & 4 & 4 & 4 & 4 & 4 \\
\hline 40 & 4 & 4 & MS & 4 & 4 & 0 & NT \\
\hline 41 & 4 & 4 & 4 & 4 & 4 & 4 & 4 \\
\hline 42 & 4 & 4 & $\mathrm{NC}$ & 4 & 4 & 4 & 4 \\
\hline 43 & 4 & 4 & NT & 4 & $\mathrm{NC}$ & 4 & 4 \\
\hline 44 & 4 & 3 & 4 & 4 & 4 & 4 & 4 \\
\hline
\end{tabular}

NT, non-tumor cells; MS, missing disc; NC, necrotic tumor cells only.

the biopsy discs taken from specimens with positive p53 staining, $112 / 138$, or $81 \%$, were given a score suggesting positive p53 staining. The remaining $19 \%$ were either false negatives or could not be assigned a score.

The 8 false-positive discs came from seven different tumor specimens. Different paraffin blocks from each of these specimens were pulled, sec- tioned, and stained with p53. These were again all scored as 0 , meaning $<2 \%$ of the tumor cells stained positive for p53.

Table 3 shows the distribution of whole specimens and their resulting disc samples by score ranges. The number of discs in each score range is shown. Of note, a large majority of discs are grouped along the diagonal (shown in bold), indicating concordance between the disc score and the whole-specimen score.

Based on these data, probabilities were calculated to estimate the chance that one, two, three, and four randomly chosen biopsy discs would correctly identify positive or negative staining in the whole-tissue specimen (Table 4). The mean probability of one randomly chosen disc correctly identifying the presence or absence of p53 staining in the whole specimen is 0.78 with a $95 \%$ confidence interval of $0.72-0.85$. Using four discs, the mean probability is 0.97 with the first confidence interval to reach $1.00(0.93-1.00)$.

\section{DISCUSSION}

Tissue microarray technology, although a powerful analytic tool, requires that an early question for each tumor type be answered: Do the samples adequately represent the whole specimens from which they were taken? This question is paramount when studying epithelial tumors with significant intratumoral heterogeneity. Previous studies have suggested that taking two biopsy cores $0.6 \mathrm{~mm}$ in diameter is sufficient for immunohistochemical studies (6). Although this may be sufficient when studying homogeneous tumors, it is not so if there is a significant amount of variability in the staining patterns of the tumor cells. Furthermore, many of the discs do not contain tumor cells, and if only two biopsies were taken, many specimens would be represented on the array with only one biopsy disc.

Merseburger et al. (17) reported on the use of tissue microarray technology in prostate carcinoma from radical prostatectomy specimens. Using only two biopsy cores per specimen, they reported $43 \%$ p53 overexpression using the microarray technology compared with $66 \%$ from whole-slide radical

TABLE 3. Distribution of Disc Scores

\begin{tabular}{|c|c|c|c|c|c|c|c|c|c|}
\hline \multirow{2}{*}{$\begin{array}{c}\text { Whole Section } \\
\text { Scores }\end{array}$} & \multicolumn{6}{|c|}{ Disc Scores } & \multicolumn{3}{|c|}{ Unscorable } \\
\hline & $0-0.3$ & $\begin{array}{c}0.67- \\
1.3\end{array}$ & $\begin{array}{c}1.67- \\
2.3\end{array}$ & $2.67-3.3$ & $3.67-4$ & $\begin{array}{c}\text { Total } \\
\text { Scored }\end{array}$ & $\begin{array}{c}\text { No } \\
\text { Tumor }\end{array}$ & Missing & Necrotic \\
\hline $0(\mathrm{n}=21)$ & 95 & 8 & 0 & 0 & 0 & 103 & 16 & 2 & 5 \\
\hline $0.67-1.3(n=4)$ & 1 & 5 & 7 & 2 & 0 & 15 & 7 & 0 & 2 \\
\hline $1.67-2.3(\mathrm{n}=5)$ & 1 & 7 & 14 & 5 & 1 & 28 & 2 & 0 & 0 \\
\hline $2.67-3.3(\mathrm{n}=5)$ & 0 & 0 & 2 & 7 & 18 & 27 & 2 & 0 & 1 \\
\hline $3.67-4(\mathrm{n}=9)$ & 1 & 0 & 0 & 5 & 39 & 45 & 4 & 1 & 4 \\
\hline NT $(n=2)$ & & & & & & & 10 & 1 & 1 \\
\hline
\end{tabular}


TABLE 4. Probabilities of Discs Predicting Whole Specimen Score

\begin{tabular}{lcc}
\hline Number of Discs & Mean Probability & $95 \%$ CI \\
\hline 1 & 0.78 & $0.72-0.85$ \\
2 & 0.89 & $0.84-0.94$ \\
3 & 0.94 & $0.90-0.99$ \\
4 & 0.97 & $0.93-1.00$ \\
5 & 0.97 & $0.93-1.00$ \\
6 & 1.00 & $1.00-1.00$ \\
\hline
\end{tabular}

prostatectomy specimens. Those investigators conclude that their study shows the limitations of TMA technology but also concede that "additional samples from each tumor may have resulted in a greater number of positive cases." These findings reinforce our conclusions that two biopsy cores do not adequately represent the whole specimen in epithelial tumors with significant heterogeneity.

Our data indicate that four or more biopsy cores $0.6 \mathrm{~mm}$ in diameter should be taken from each laryngeal carcinoma specimen. The reasons for this are several. First, $17 \%$ of biopsy discs in our series did not contain tumor (discussed below). Also, the chance of one biopsy disc accurately representing the whole specimen in this study was $78 \%$. This is due to both the intratumor variability of the staining for p53 and the false-negative and -positive rates. There were many tumors with a wholespecimen score of $1-2$, indicating that $<50 \%$ and as low as $2 \%$ of the tumor cells present stained positive for $\mathrm{p} 53$.

Eight discs from seven different tumor specimens were scored as false positives (positive disc with negative whole section). To determine whether these discs were indeed false positives or whether they were true positives and simply represented tumor heterogeneity, multiple paraffin blocks from these seven specimens were sectioned. Slides were stained for p53, and all slides were scored as 0 , indicating that $<2 \%$ of the tumor cells stained for p53. Thus, these positive discs, by our criteria, indeed represented false positives. Such false positives may occur because of lab error, or increased background staining around those discs. Sampling error may also account for false positives. For instance, the few cells that stained positive were captured in the $0.6-\mathrm{mm}$ disc, but considered on the whole slide, they constituted $<2 \%$ of the tumor cells.

A significant number of core biopsies (17\%) did not contain viable tumor cells and therefore were unable to be scored in the immunohistochemical analysis. This percentage is almost identical to that reported by others (8). Figure 2 shows a specimen that was given an average score of 3 on whole section, but two of the six cores were not given numerical scores. There are three reasons that some discs did not receive a numerical score: first, the biopsy may contain only necrotic tumor cells with pyknotic nuclei (see Fig. 2, Panel G). This accounted for $30 \%$ of these unscorable discs. Second, the biopsy disc itself may be completely missing, secondary to technical error while making the array (accounting for three discs). Finally, 52\% of the biopsies that could not be scored contain only nontumor cells, such as fatty or fibroblastic tissue around or within the tumor (see Fig. 2, Panel C).

To decrease the number of unscorable discs, technical modifications may be made. Previously reporting investigators advocate examining each specimen microscopically before taking the core biopsies and marking on the slide areas of tumor (8). We did not take this step and report a similar rate of unscorable biopsies. Instead, we grossly inspected the specimen and took the biopsies from apparent nonnecrotic portions of the tumor. However, in specimens in which the presence of tumor is not clear by gross inspection, we do advocate looking at an H\&E slide from the whole specimen to identify areas of tumor.

In conclusion, when making tissue microarrays with squamous cell carcinoma of the larynx, we advocate taking four biopsy cores from each specimen. This compensates for the intratumor heterogeneity of these tumors and represents the whole specimen at a high probability. Such tissue microarrays will allow a rapid and powerful approach to immunohistochemistry studies in squamous cell cancers of the head and neck.

Acknowledgments: The authors thank Justine Ritchie, Department of Biostatistics at the University of Iowa for statistical consultation and also thank the University of Iowa Pathology Core Lab, Patricia Palecek, Christine Bromley, Jan Rodgers, and Richard Rodgers, for their invaluable assistance.

\section{REFERENCES}

1. Wan WH, Furtuna MB, Furmanski P. A rapid and efficient method for testing immunohistochemical reactivity of monoclonal antibodies against multiple tissue samples simultaneously. J Immunol Methods 1987;103:121-9.

2. Kononen J, Bubendorf L, Kallioniemi A, Barlund M, Schraml $\mathrm{P}$, Leighton $\mathrm{S}$, et al. Tissue microarrays for high-throughput molecular profiling of tumor specimens. Nat Med 1998;7: 844-7.

3. Rimm DL, Camp RL, Charette LA, Costa J, Olsen DA, Reiss M. Tissue microarray: a new technology for amplification of tissue resources. Cancer J 2001;7:24-31.

4. Rubin MA, Dunn R, Strawderman M, Rienta KJ. Tissue microarray sampling strategy for prostate cancer biomarker analysis. Am J Surg Pathol 2002;26:312-9.

5. Milanes-Yearsley M, Hammond EH, Pajak TF, Cooper JS, Chang C, Griffin T, et al. Tissue micro-array: a cost and time-effective method for correlative studies by regional and national cancer study groups. Mod Pathol 2002;15:1366-73.

6. Kuwabara S, Ajioda Y, Watanabe H, Hitomi J, Nishikura K, Hatakeyama K. Heterogeneity of p53 mutational status in 
esophageal squamous cell carcinoma. Jpn J Canc Res 1998; 89:405-10.

7. Baisse B, Bouzourene H, Saraga EP, Bosman FT, Benhattar J. Intratumor genetic heterogeneity in advanced human colorectal adenocarcinoma. Int J Cancer 2001;93:346-52.

8. Camp RL, Charette LA, Rimm DL. Validation of tissue microarray technology in breast carcinoma. Lab Invest 2000; 80:1943-9.

9. Hoos A, Urist MJ, Stojadinovic A, Mastorides S, Dudas ME, Leung DHY, et al. Validation of tissue microarrays for immunohistochemical profiling of cancer specimens using the example of human fibroblastic tumors. Am J Pathol 2001; 158:1245-51.

10. Fernebro E, Dictor M, Bendahl PO, Ferno M, Nilbert M. Evaluation of the tissue microarray technique for immunohistochemical analysis in rectal cancer. Arch Pathol Lab Med 2002;126:702-5.

11. Engellau J, Akerman M, Anderson H, Domanski HA, Rambech E, Alvegard TA, et al. Tissue microarray technique in soft tissue sarcoma: immunohistochemical Ki-67 expression in malignant fibrous histiocytoma. Appl Immunohistochem Mol Morphol 2001;9:358-63.
12. Greenlee RT, Hill-Harmon MB, Murray T, Thun M. Cancer Statistics. CA Cancer J Clin 2001;51:15-36.

13. Bradford CR, Qhu S, Wolf GT, Poore J, Fisher SG, Beals T, et al. Overexpression of $\mathrm{P} 53$ predicts organ preservation using induction chemotherapy and radiation in patients with advanced laryngeal cancer. Otolarynol Head Neck Surg 1995; 113:408-12.

14. Uhlman DL, Adams G, Knapp D, Aepplli DM, Niehans G. Immunohistochemical staining for markers of future neoplastic progression in the larynx. Cancer Res 1996;56:2199-205.

15. Mousses S, Bubendorf L, Wagner U, Hostetter G, Kononen J, Cornelison $\mathrm{R}$, et al. Clinical validation of candidate genes associated with prostate cancer progression in the CWR22 model system using tissue microarrays. Cancer Res 2002;62: $1256-60$.

16. Rosner B, editor. Fundamentals of biostatistics. 5th ed. Pacific Grove, CA: Duxbury; 2000.

17. Merseburger AS, Kuczyk MA, Serth J, Bokemeyer C, Young DY, Sun L, et al. Limitations of tissue microarrays in the evaluation of focal alterations of bcl-2 and p53 in whole mount derived prostate tissues. Oncol Rep 2003;10:223-8. 